\title{
Supramolecular nanoparticles generated by the self-assembly of polyrotaxanes for antitumor drug delivery
}

This article was published in the following Dove Press journal:

International Journal of Nanomedicine

4 October 2012

Number of times this article has been viewed

\author{
Rong Liu',2,* \\ Yusi Lai ${ }^{1, *}$ \\ Bin $\mathrm{He}^{1}$ \\ Yuan $\mathrm{Li}^{\prime}$ \\ Gang Wang' \\ Shuang Chang' \\ Zhongwei Gu' \\ 'National Engineering Research \\ Center for Biomaterials, Sichuan \\ University, Chengdu, China; ${ }^{2}$ Dalian \\ Institute of Chemical Physics, Chinese \\ Academy of Sciences, Dalian, China \\ *These authors contributed equally \\ to this paper
}

Correspondence: Bin $\mathrm{He}$ National Engineering Research Center for Biomaterials, Sichuan University, 29\# Wangjiang Road, Chengdu 610064, China

Tel +86 28 854I 2923

Fax +86 $28854 \mid 0653$

Email bhe@scu.edu.cn

Zhongwei Gu

National Engineering Research Center for Biomaterials, Sichuan University, 29\# Wangjiang Road, Chengdu 610064, China

Tel +862885410336

Fax +86 28 854I 0653

Email zwgu@scu.edu.cn

\begin{abstract}
A new approach of fabricating supramolecular nanoparticles generated by selfassembly polyrotaxanes for antitumor drug delivery has been reported. Cinnamic-acid-modified poly(ethylene glycol) chains were threaded in $\alpha$-cyclodextrins to form polyrotaxanes. The polyrotaxanes self-assembled supramolecular nanoparticles. The morphology of the nanoparticles was changed from nanovesicle to micelle after the antitumor drug, doxorubicin, was loaded. The release profile of the drug-loaded nanoparticles was investigated, and it was found that the sustaining release time could last for 32 hours. The drug-loaded nanoparticles were co-cultured with mouse $4 \mathrm{~T} 1$ breast cancer cells with a drug concentration of $10 \mu \mathrm{g} / \mathrm{mL}$; the cell survival rate was 3.3\% after a 72-hour incubation. In an in vivo study of breast cancer in a mouse model, the drug-loaded nanoparticles were injected in the tail veins of mice with a dose of $5 \mathrm{mg} / \mathrm{kg}$ body weight. The tumor inhibition rate of drug-loaded nanoparticles was $53 \%$, which was better than that of doxorubicin hydrochloride. The cardiac toxicity of doxorubicin was decreased greatly after the encapsulation into supramolecular polyrotaxane nanoparticles.
\end{abstract}

Keywords: polyrotaxane, self-assembly, nanoparticle, doxorubicin, supermolecular

\section{Introduction}

In cancer chemotherapy, nanoparticles exhibit unique advantages in escaping the capture of the reticuloendothelial system, ${ }^{1}$ and they also achieve long circulation in the blood stream to passively target tumor tissues due to their enhanced permeability and retention..$^{2-5}$ Thus, nanoparticles attract much interest from chemists and materials scientists for antitumor drug delivery. Nanospheres, micelles, and nanogels have been extensively reported ${ }^{1,2}$ as being drug carriers via the fabrication approaches of oil/ water emulsification, amphiphilic self-assembly, and microemulsion polymerization. As the antitumor drugs are physically encapsulated in these nanoparticles, low drugloading content and encapsulation efficiency are some of the problems faced in drug delivery. ${ }^{6-9}$ Many strategies have been carried out to improve the drug-loading content. Mesoporous silicon nanoparticles, graphene, and carbon nanotubes have been used to load drugs due to their high specific surface area and for their ability to adsorb a large amount of drugs. ${ }^{10-12}$ The unavoidable drawbacks of these inorganic nanoparticles are the fact that they are undegradable and they do not circulate for a long time.

Host-guest interaction is an effective approach in fabricating nanoparticles. ${ }^{13-16}$ Polyrotaxanes are necklace-like inclusion complexes formed by the host-guest interaction between cyclodextrins and polymeric chains via the threading of polymeric chains in the cavity of cyclodextrins. ${ }^{17-20}$ With the regular stack of cyclodextrins along polymeric chains, nanoparticles are self-assembled by polyrotaxanes. Moreover, $\alpha$-cyclodextrin $(\alpha-C D)$ 
is a cyclic oligosaccharide composed of six D-glucose units linked by $1,4-\alpha$-glucosidic bonds; it is widely used in pharmaceutical science for its excellent biocompatibility. Poly(ethylene glycol) (PEG) is regarded as biodegradable material when its molecular weight is lower than 10,000 Dalton, because it is eliminated by the kidney. With this in mind, it is evident that the $\alpha-C D / P E G$ polyrotaxane nanoparticles are attractive drug carriers. Taking the advantages of the hydroxyl functional groups on cyclodextrins into account, cationic segments are immobilized and the polyrotaxanes can be used as nonviral gene vectors. The modified polyrotaxanes can condense DNA efficiently and exhibit excellent gene transfection activity. ${ }^{21,22}$ The fabrication of nanoparticles via the crystallization of polyrotaxanes has been reported, ${ }^{23-25}$ and it is evident that these nanoparticles are compact and cannot trap drugs inside.

Herein, we report a new strategy of fabricating polyrotaxane nanoparticles to encapsulate antitumor drugs. Hydrophobic molecule cinnamic acids were immobilized on the terminal groups of PEG $(\mathrm{Mn}=2000)$. The cinnamate terminated PEG was threaded in $\alpha-C D s$ to form polyrotaxanes. The cinnamate moieties exerted two functions: one was to decrease the number of $\alpha-C D$ in PEG chains to avoid the formation of compact nanoparticles; the other was to provide hydrophobicity in order to trap the hydrophilic antitumor drug inside. Antitumor doxorubicin was used to investigate the release properties of the polyrotaxane nanoparticles. The morphology, in vitro, and in vivo antitumor activities of the drug-loaded polyrotaxane nanoparticles are studied in detail.

\section{Materials and methods \\ Materials}

PEG $(\mathrm{Mn}=2000 \mathrm{~g} / \mathrm{mol})$ and $\alpha-\mathrm{CD}$ were purchased from Sigma-Aldrich (St Louis, MO) and vacuum-dried at room temperature for 24 hours before use. N, $N^{\prime}$ dicyclohexylcarbodiimide (DCC), cinnamic acid, and dimethyl sulfoxide (DMSO)-d $\mathrm{d}_{6}$ were purchased from Sigma-Aldrich and used as received. Doxorubicin hydrochloride (Beijing Zhongshuo Pharmaceutical Technology Development Co, Ltd, Beijing, China) was deprotonated in water, and the $\mathrm{pH}$ value was adjusted to 9.6 to obtain doxorubicin. ${ }^{26}$ Dulbecco's modified Eagle's medium (DMEM), $100 \times$ mycillin, and fetal bovine serum were purchased from Life Technologies Co, (Carlsbad, CA) and used for the cytotoxicity test. Cell lines NIH/3T3 (mouse embryonic fibroblast cell) and 4T1 (mouse breast cancer cell) were obtained from the Chinese Academy of Science Cell Bank for Type Culture Collection (Shanghai, China). All other chemicals were purchased from Kelong Chemical Co (Chengdu, China) and used as received.

\section{Measurements}

${ }^{1} \mathrm{H}$ NMR spectra were performed on a Bruker av-400 spectrometer (Bruker Corporation, Billerica, MA) using DMSO- $\mathrm{d}_{6}$ as solvents. Transcranial magnetic stimulation was used as the internal reference. Ultrasound was performed on ultrasonic cleaner KQ-300DE (Kunshan Ultrasonic Instruments Co, Ltd, Kunshan City, China) at a 70\% frequency. $\mathrm{X}$-ray diffractometry (XRD) patterns were obtained at room temperature on an X'Pert pro MPD X-ray diffractometer (PANalytical BV, Almelo, the Netherlands), and the powder samples were mounted on a sample holder and scanned from $5^{\circ}$ to $50^{\circ}$. Ultraviolet-visible spectra were recorded on Lambda 650 (PerkinElmer Inc, Waltham, MA) in a range from $200 \mathrm{~nm}$ to $500 \mathrm{~nm}$. Dynamic light scattering (DLS) experiments were performed on a Malvern Zetasizer Nano ZS (Malvern Instruments Ltd, Malvern, UK) at an angle of $90^{\circ}$ at $25^{\circ} \mathrm{C}$. Transmission electron microscopy (TEM) was performed on a Hitachi H600-4 (Hitachi Ltd, Tokyo, Japan). Atomic force microscopy (AFM) imaging was performed using an MFP3D probe (Asylum Research, Santa Barbara, $\mathrm{CA}$ ) in tapping mode. Cellular uptake was examined by confocal laser scanning microscopy (Leica TCP SP5). Enzymelinked immunosorbent assay analysis was performed on enzyme-linked immunosorbent assay instrument Model-550 (Bio-Rad Co, Hercules, CA).

\section{Synthesis of cinnamic-acid-modified PEG (Cin-PEG)}

A total of $20 \mathrm{~g}$ of PEG $(\mathrm{Mn}=2000)$ and $4.44 \mathrm{~g}$ of cinnamic acid were dissolved in $200 \mathrm{~mL}$ of methylene chloride; $7.98 \mathrm{~g}$ of DCC dissolved in $100 \mathrm{~mL}$ methylene chloride was slowly dripped into the mixture in an ice bath. After the DCC solution was dripped into the ice bath, the mixture was stirred in the dark at room temperature for 24 hours. The insoluble $\mathrm{N}, \mathrm{N}$-dicyclohexylurea was filtered off. The filtrate was concentrated in a rotary evaporator. The modified PEG was precipitated from the concentrate filtrate by the addition of excessive diethyl ether. The product was filtered, washed with water, and dried in a vacuum.

\section{Preparation of polyrotaxanes}

To prepare the polyrotaxanes, $1 \mathrm{~g}$ of Cin-PEG was dissolved in $20 \mathrm{~mL}$ of distilled water, and the solution was added to $10 \mathrm{~mL}$ of saturated aqueous solution of $\alpha$-CDs at room temperature. The mixture was ultrasonically agitated for 10 minutes and then allowed to stand overnight in a refrigerator at $4^{\circ} \mathrm{C}$ to precipitate white paste. The product was collected by centrifugation, and then was washed with water three times 
to remove the unthreaded $\alpha-C D$ s and PEG. The white paste precipitate was dried in a vacuum at room temperature to yield the polyrotaxanes. This process was performed in the dark.

\section{Biocompatibility test}

Mouse NIH 3 T3 fibroblasts were cultured in DMEM at $37^{\circ} \mathrm{C}$ with $5 \% \mathrm{CO}_{2}$. The $3 \mathrm{~T} 3$ fibroblasts were harvested in a logarithmic growth phase, seeded on 96 wells at a cellular density of $1 \times 10^{3}$ cells/well, and incubated for 1 day. Polyrotaxanes were dissolved and diluted in PBS buffer to give final concentrations ranged from $5.4 \times 10^{-3}$ to $0.54 \mathrm{mg} / \mathrm{mL}$. The polyrotaxanes and fibroblasts were incubated for 30 hours. The CCK-8 assay at $490 \mathrm{~nm}$ was performed and the percentage of cell viability was then determined.

\section{Preparation of drug-loaded polyrotaxane nanoparticles}

Drug-loaded nanoparticles were prepared by concentrate centrifugation. The polyrotaxanes $(10 \mathrm{mg})$ were dissolved in $5 \mathrm{~mL}$ water, and stirred for 5 minutes. Doxorubicin $(2.5 \mathrm{mg})$ was dissolved in $1 \mathrm{~mL}$ tetrahydrofuran (THF). The solution was dripped into the polyrotaxane solution and stirred for 24 hours. Five milliliters of water was added into the mixture after THF was volatilized. Free doxorubicin and THF were removed using concentrate centrifugation pipe with a dialysis membrane $(\mathrm{MWCO}=3000)$. The process was repeated three times. After the centrifugation, the solution was freeze-dried to get the doxorubicin-loaded polyrotaxane nanoparticles.

The doxorubicin loaded nanoparticles were dissolved in $3 \mathrm{~mL}$ of DMSO to determine the drug-loading content. The doxorubicin concentration was tested using the UV-VIS spectrophotometer at $485 \mathrm{~nm}$. The drug-loading content was calculated based on the standard curve obtained from doxorubicin in DMSO. The drug-loading content (DLC) and encapsulation efficiency (EE) were calculated according to the following formulae:

$$
\begin{aligned}
\operatorname{DLC}(\text { wt } \%)= & (\text { weight of loaded drug/weight of } \\
& \text { drug-loaded nanoparticles }) \times 100 \% \\
\text { EE }(\%)= & (\text { weight of loaded drug } / \text { weight of } \\
& \text { drug in feeding }) \times 100 \%
\end{aligned}
$$

\section{In vitro drug release}

The drug-loaded nanoparticles were dispersed and diluted in phosphate-buffered saline (PBS) solution to yield a final concentration of $2 \mathrm{mg} / \mathrm{mL}$. The diluted solution $(0.5 \mathrm{~mL})$ was transferred to dialysis membrane tubes $(\mathrm{MWCO}=3000)$. The tubes were then immersed in a flask containing $25 \mathrm{~mL}$ of PBS solution $(\mathrm{pH}=7.4)$ and shaken at a speed of $100 \mathrm{rev} / \mathrm{min}$ at $37^{\circ} \mathrm{C}$. At specific time intervals, $1 \mathrm{~mL}$ of solution was withdrawn from the release medium and replaced with fresh PBS solution.

\section{Cellular uptake}

Mouse 4T1 breast cancer cells were cultured in DMEM with $10 \%$ fetal bovine serum at $37^{\circ} \mathrm{C}$ in a $5 \% \mathrm{CO}_{2}$ environment. The 4T1 cells at logarithm phase were seeded on a 6-well culture dish at a cell density of $1 \times 10^{4}$ cells/well. After 1 day, free doxorubicin hydrochloride and doxorubicin loaded nanoparticles were dispersed and diluted in PBS to give the final doxorubicin concentration of $10 \mu \mathrm{g} / \mathrm{mL}$, which were then added into the plates. After being separately incubated for 3 and 13 hours, the cells were thoroughly washed with PBS three times and visualized under confocal laser scanning microscopy (Leica TCP SP5). Doxorubicin was excited at $485 \mathrm{~nm}$ with emission at $595 \mathrm{~nm}$.

\section{In vitro inhibition efficacy}

The 4T1 cells at logarithm phase were seeded on a 96-well culture dish at a cell density of $1 \times 10^{3}$ cells/well. After being incubated for 1 day, free doxorubicin hydrochloride and doxorubicin-loaded nanoparticles were dispersed and diluted in PBS solution to give the final doxorubicin concentration of $10 \mu \mathrm{g} / \mathrm{mL}$. The cells were incubated with free doxorubicin hydrochloride and doxorubicin-loaded nanoparticles for 72 hours. The CCK- 8 assay at $490 \mathrm{~nm}$ was performed at $0,12,24,36,48,60$, and 72 hours. The percentage of cell viability was then determined. Five parallel samples in each specimen were used for testing.

\section{In vivo antitumor study}

Permission to conduct the experiment on animals was obtained from the ethics committee of Sichuan University (Sichuan, China). A total of $48 \mathrm{BALB} / \mathrm{c}$ mice (male, body weight: $21-24 \mathrm{~g}$ ) purchased from the West China Experimental Animal Center of Sichuan University (Sichuan, China) were maintained in a germ-free environment and allowed free access to food and water. All animal experiments were performed according to the institutional and National Institutes of Health guidelines for the care and use of research animals.

During the experiment, $5 \times 10^{5} 4 \mathrm{~T} 1$ cells were subcutaneously injected into the left flanks of BALB/c mice. After the inoculated tumor volume reached $100-200 \mathrm{~mm}^{3}$, the mice were randomly divided into four groups. Each formulation was injected intravenously via the tail vein with a dose of 
$5 \mathrm{mg}$ of doxorubicin per $\mathrm{kg}$ of body weight. Four injections were taken at 4-day intervals. The tumor volume was monitored at prescribed time intervals. The tumor volume was calculated using the formula given below:

$$
\mathrm{V}\left[\mathrm{mm}^{3}\right]=1 / 2 \times \mathrm{LW}^{2}
$$

where $\mathrm{L}$ and $\mathrm{W}$ represent the long and short diameters of the tumor tissues. On the 19th day after treatment, all mice were sacrificed. The difference in tumor growth was evaluated using the $t$-test and was considered significant if $P<0.05$.

\section{Pathological examination}

Sections of the hearts of sacrificed mice treated with doxorubicin hydrochloride and doxorubicin loaded polyrotaxane nanoparticles were fixed in $10 \%$ buffered formaldehyde, embedded in paraffin, and then sectioned. The sections were then stained routinely with hematoxylin-eosin for histological assessment.

\section{Results and discussion}

The structures of Cin-PEG, $\alpha$-cyclodextrin, and corresponding polyrotaxane were characterized by ${ }^{1} \mathrm{H}$ NMR (Figure 1). The protons of $\mathrm{CH}_{2} \mathrm{CH}_{2} \mathrm{O}$ in the $\mathrm{PEG}$ chain appeared at $\delta=3.6 \mathrm{ppm}$. The terminal cinnamate groups changed the chemical environment of the EG (ethylene glycol) unit connected to cinnamate and thus led to a signal shift to $\delta=3.7$ and $4.3 \mathrm{ppm}$, respectively. The protons in cinnamate were localized from $\delta=6$ to $8 \mathrm{ppm}$. The signals of protons in $\mathrm{CH}=\mathrm{CH}$ were split and appeared at around $\delta=7.7 \mathrm{ppm}$ and $\delta=6.5 \mathrm{ppm}$. The signals of protons in the benzene ring appeared in two sites; the peak at $\delta=7.4$ ppm was attributed to the protons in ortho and para positions. The signal at $\delta=7.5 \mathrm{ppm}$ was assigned to the

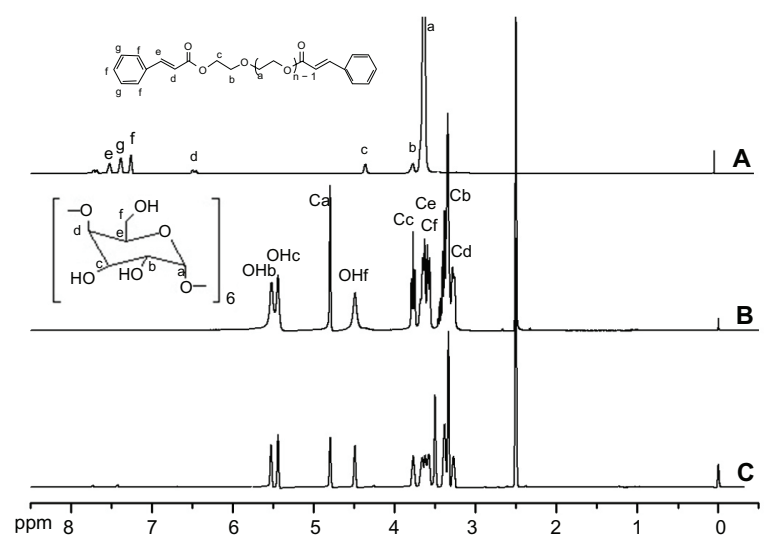

Figure I The 'H NMR spectra of cinnamic-acid-modified PEG (A), $\alpha-C D(B)$, and polyrotaxanes (C).

Abbreviations: PEG, poly(ethylene glycol); $\alpha-C D, \alpha$-cyclodextrin. protons in meta position. The integrity ratio of $\mathrm{COOCH}_{2} \mathrm{CH}_{2} \mathrm{O}$ (Figure 1Ac) to $\mathrm{OCH}_{2} \mathrm{CH}_{2} \mathrm{O}$ (Figure 1Aa) was used to calculate the number of immobilized cinnamic acid. The result showed that both terminal groups of PEG were immobilized with cinnamic acid. The spectrum of polyrotaxane is presented in Figure $1 \mathrm{C}$. The proton signals of $\alpha$-cyclodextrin in the spectrum of polyrotaxane were strong. Moreover, the signal at $\delta=3.5 \mathrm{ppm}$ was noted among the $\mathrm{OCH}_{2} \mathrm{CH}_{2} \mathrm{O}$ protons in modified PEG, which shifted from $\delta=3.6 \mathrm{ppm}$ to $\delta=3.5 \mathrm{ppm}$. All the protons in PEG and $\alpha$-CDs were detected in the spectrum, which suggested that the polyrotaxane was formed. The mole ratio of EG/CD was calculated from the integrities of $\mathrm{OCH}_{2} \mathrm{CH}_{2}$ in modified PEG and $\mathrm{CH}(\mathrm{Ca})$ in $\alpha-\mathrm{CDs}$. The calculated $\mathrm{EG} / \mathrm{CD}$ was 2.8 , which meant that the average numbers of $\alpha-C D$ molecules in a Cin-PEG chain were 16 as the repeated units of EG in PEG $(\mathrm{Mn}=2000)$ were about 44. The ratio of $\mathrm{EG} / \mathrm{CD}$ in $\alpha-\mathrm{CD} / \mathrm{PEG}$ polypseudorotaxane was $2.0-2.1 ;^{27,28}$ the higher ratio of EG/CD implied that the terminal cinnamates in the PEG chains reduced the numbers of $\alpha$-cyclodextrins in PEG chains. It was favorable for weakening the crystallization of polyrotaxane to avoid the formation of compact nanoparticles.

$\mathrm{XRD}$ is a powerful tool for confirming the formation of polyrotaxane. The XRD spectra of $\alpha$-cyclodextrin, Cin-PEG, and polyrotaxane are presented in Figure 2. Strong crystallization was observed in the spectrum of $\alpha$-cyclodextrin (Figure 2A). Cin-PEG also exhibited strong crystallinity, and the prominent peaks of PEG crystal appeared at $2 \theta=19.3^{\circ}$ and $23.4^{\circ}$ (Figure $2 \mathrm{~B}$ ). In the spectra of polyrotaxane, a very strong peak occurred at $2 \theta=19.7^{\circ}$ and was presented in the diffraction patterns (Figure 2C). It was the characteristic peak associated with a channel-type crystalline structure by virtue of the polymeric nature of the guest molecules. ${ }^{17-20,29}$

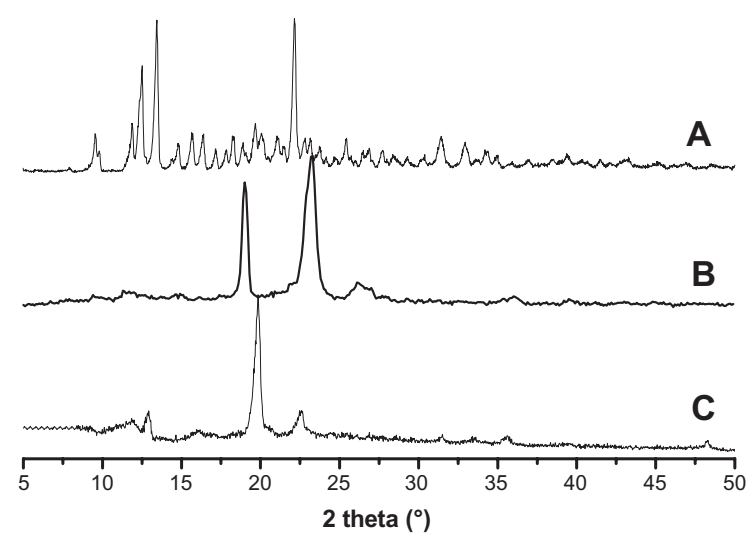

Figure 2 The XRD spectra of $\alpha$-cyclodextrin (A), cinnamic-acid-modified PEG (B), and polyrotaxane (C).

Abbreviations: XRD, X-ray diffractometry; PEG, poly(ethylene glycol). 
The XRD result demonstrated that the Cin-PEG was threaded in $\alpha$-CDs to form necklace-like polyrotaxanes.

Doxorubicin was trapped in the polyrotaxane nanoparticles. The morphologies of the blank and drug-loaded polyrotaxane nanoparticles were characterized by DLS, TEM, and AFM (Figure 3). The DLS results showed that both the blank and drug-loaded nanoparticles were monodispersed and the mean diameters were 57 and 107 nanometers, respectively. The diameter of the nanoparticles was increased after drug encapsulation (Figure 3A and B). The TEM photograph of the blank nanoparticles clearly showed that the nanoparticles had a core-shell structure and the shell layer was darker, which meant that this layer was compact (Figure 3C). The AFM image of the blank nanoparticles supported this conclusion. The height of the blank nanoparticles was measured by AFM using the tapping mode. The nanoparticles were sunk inside and the height of the nanoparticles was about 0.8 nanometers, which was nearly the same height as one $\alpha$-cyclodextrin. ${ }^{30,31}$ The wall
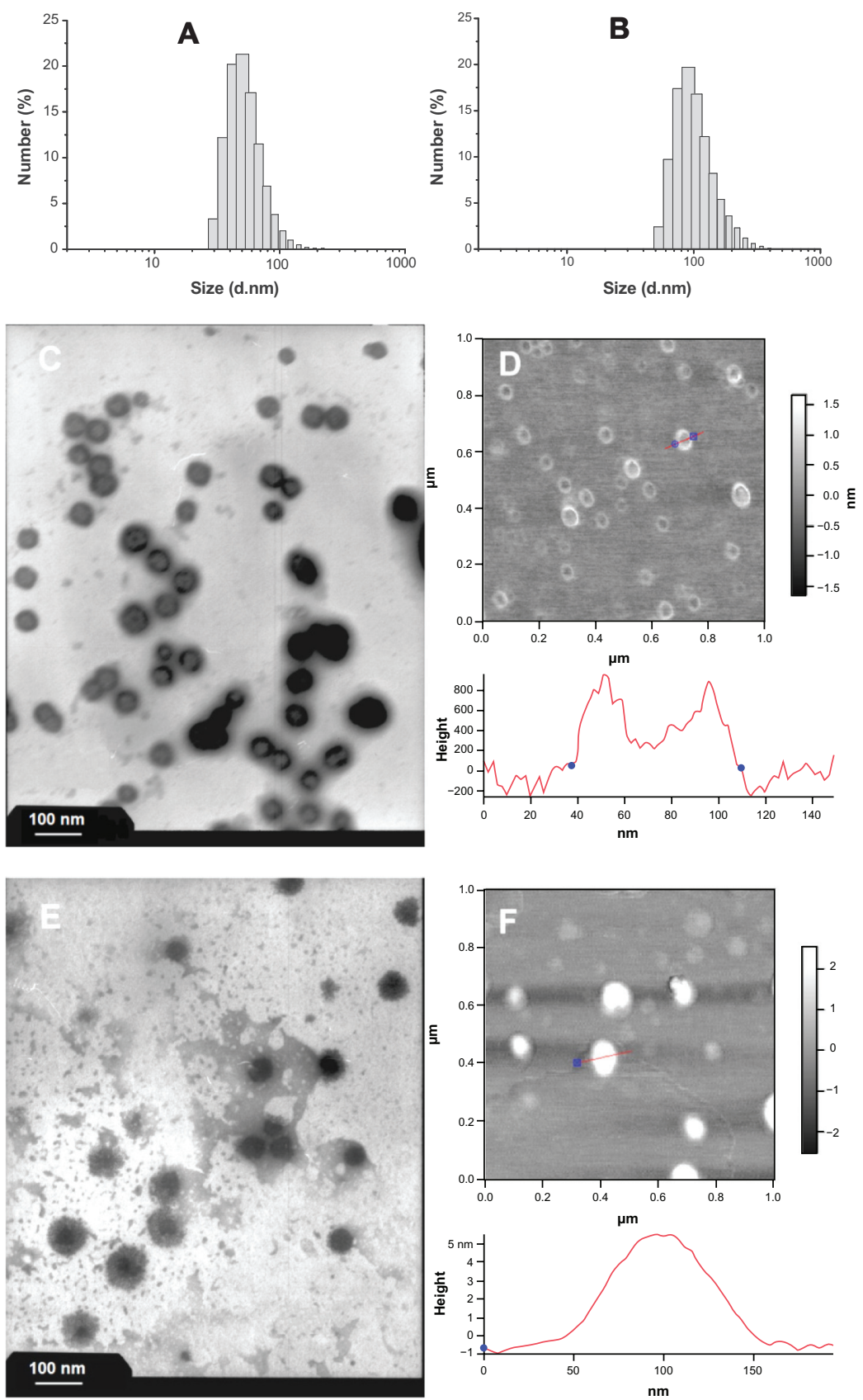

Figure 3 Size distributions and morphologies of blank and drug-loaded polyrotaxane nanoparticles. (A) DLS of blank nanoparticles; (B) DLS of drug-loaded nanoparticles; (C) TEM photograph of blank nanoparticles; (D) AFM image of blank nanoparticles; (E) TEM photograph of drug-loaded nanoparticles; (F) AFM image of drug-loaded nanoparticles.

Abbreviations: DLS, dynamic light scattering; TEM, transmission electron microscopy; AFM, atomic force microscopy. 
thickness of the sunk nanoparticles measured by AFM was about 20 nanometers (Figure 3D). Both TEM and AFM images demonstrated that the blank polyrotaxane nanoparticles were hollow with vesicle-like morphology. Interestingly, the morphology of drug-loaded nanoparticles changed significantly; it was still core-shell structured, but the core was dark and the shell layer was light in the TEM photograph (Figure 3E). The compact layer was transferred from the shell to the core after the drug was loaded; the shell thickness of the drug-loaded nanoparticles measured from TEM photograph was about 35 nanometers (Figure 3E). The height of the nanoparticles measured by AFM was more than 5 nanometers and no sinking was observed in the nanoparticles (Figure 3F). The results of both TEM and AFM proved that the drug-loaded nanoparticles were no longer nanovesicles but micelle-like nanoparticles.

The hypothesized formation process of the blank and drug-loaded polyrotaxane nanoparticles was illustrated in Figure 4. Cinnamic acid is a hydrophobic molecule, and the polyrotaxanes with Cin-PEG chains are amphiphiles. The amphiphiles formed vesicle-like hollow polymersomes. Once the hydrophobic drug doxorubicin was trapped, the selfassembly of polyrotaxanes was changed. The doxorubicin was centered at the core to gather the hydrophobic segments in the polyrotaxanes, thus, the polyrotaxanes self-assembled micelle-like nanoparticles.

The drug-loading content and encapsulation efficiency of the polyrotaxane nanoparticles were tested and summarized in Table 1. Drug-loading content and encapsulation efficiency are important parameters in evaluating the usability of nanoparticles for antitumor drug delivery. A main problem faced by nanodrug carriers was the low drug-loading content; specifically, this property would decrease the curative effect of chemotherapy, and therefore the high dose of drugloaded nanoparticles had to be used, which increased the risk of the treatment. In most polymeric drug nanocarriers, such as nanospheres and micelles, the drug-loading content was less than $10 \% .^{6-9}$ In these polyrotaxane nanoparticles, the drug-loading content and encapsulation efficiency were

Table I The drug-loading content and encapsulation efficiency of polyrotaxane nanoparticles

\begin{tabular}{|c|c|c|c|c|c|}
\hline \multirow[t]{2}{*}{ Sample } & \multirow[t]{2}{*}{$E G / C^{a}$} & \multicolumn{2}{|c|}{$\begin{array}{l}\text { Mean diameter } \\
(\mathrm{nm})\end{array}$} & \multirow[t]{2}{*}{$\begin{array}{l}\text { DLC } \\
\text { (\%) }\end{array}$} & \multirow[t]{2}{*}{$\begin{array}{l}\text { EE } \\
\text { (\%) }\end{array}$} \\
\hline & & Before $^{b}$ & After $^{c}$ & & \\
\hline$\alpha-C D s / C i n-P E G$ & 2.8 & $57 \pm 8$ & $107 \pm 12$ & $18.4 \pm 0.7$ & 78.1 \\
\hline
\end{tabular}

Notes: aCalculated from 'H NMR spectrum; 'before drug-loading; 'after drug-loading. Abbreviations: EG, ethylene glycol; CD, cyclodextrin; DLC, drug-loading content; $\mathrm{EE}$, encapsulation efficiency; $\alpha$-CDs, $\alpha$-cyclodextrins; Cin-PEG, cinnamic-acidmodified poly(ethylene glycol).

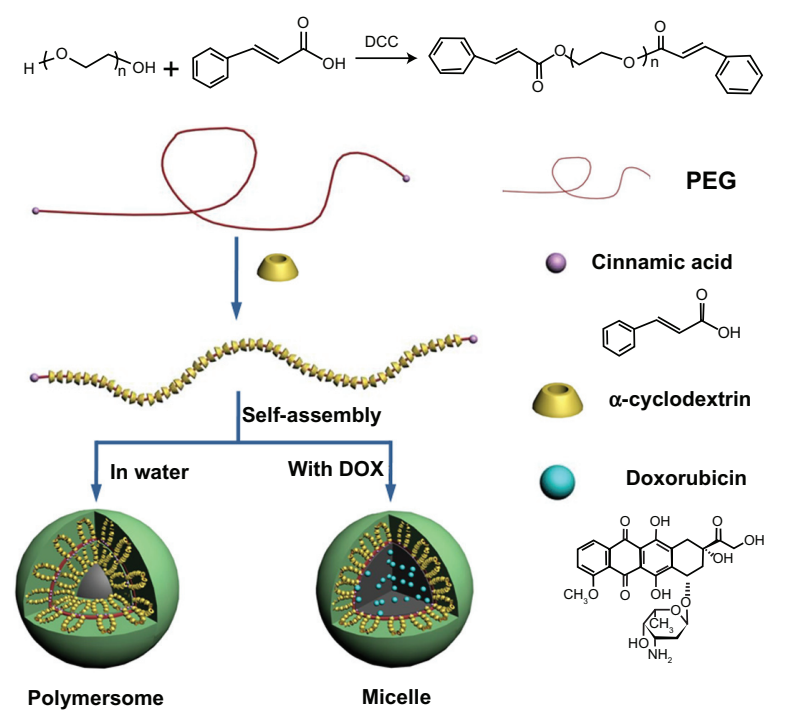

Figure 4 The illustrated formation of drug-loaded polyrotaxane nanoparticles. Abbreviations: PEG, poly(ethylene glycol); DOX, doxorubicin.

$18.4 \%$ and $78.1 \%$, respectively. The drug-loading content was much higher than that of most polymer micelles.

The release profile of drug-loaded polyrotaxane nanoparticles is presented in Figure 5. Hydrophilic doxorubicin hydrochloride was used as a control. The release of doxorubicin hydrochloride was very fast in PBS solution and the accumulated release rate was nearly $100 \%$ within the first 3 hours. For the drug-loaded nanoparticles, the burst release was about $40 \%$ in the first 2 hours. After the burst release, the sustaining release time was more than 30 hours, and the release curve was linear and nearly obeyed the rule of oneorder release (Figure 5B). The accumulated release reached about $80 \%$ when the release time was 32 hours. The release profile implied that the polyrotaxane nanoparticles were promising for doxorubicin delivery.

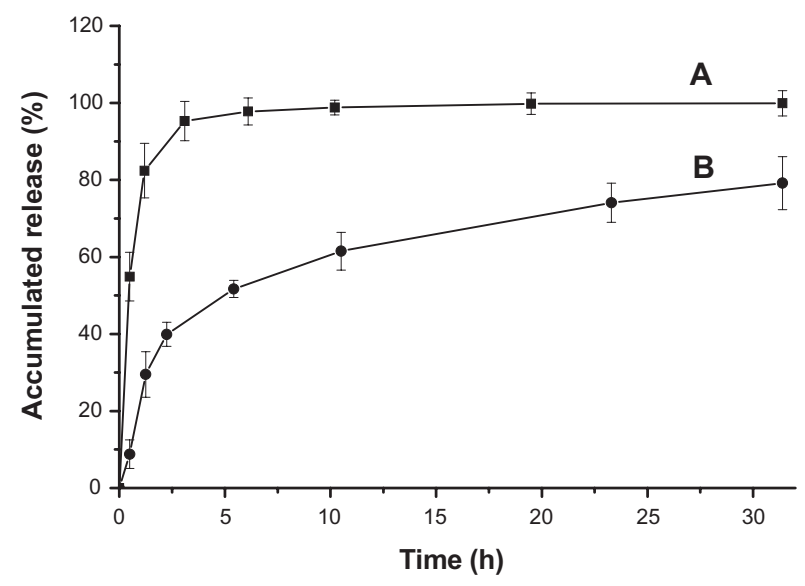

Figure 5 Release profiles of doxorubicin hydrochloride (A) and drug-loaded polyrotaxane nanoparticles (B). 
The blank polyrotaxane nanoparticles were co-cultured with mouse NIH 3T3 fibroblasts to evaluate the biocompatibility; the result is shown in Figure 6. The incubation time was 24 hours and the nanoparticle concentrations ranged from $5.4 \times 10^{-3} \mathrm{mg} / \mathrm{mL}$ to $0.54 \mathrm{mg} / \mathrm{mL}$. The cells on the cell culture plates were used as the control. The cell viability of the nanoparticles in each concentration was around $100 \%$, which demonstrated that the polyrotaxane nanoparticles were non-toxic to NIH 3T3 fibroblasts.

The drug-loaded polyrotaxanes nanoparticles were cocultured with $4 \mathrm{~T} 1$ breast cancer cells to evaluate the in vitro inhibition activity (Figure 7). The concentration of doxorubicin was $10 \mu \mathrm{g} / \mathrm{mL}$. Doxorubicin was hydrophobic, and it aggregated and precipitated in the cell culture medium, thus it could not be used as a control. Doxorubicin hydrochloride was water-soluble, so doxorubicin hydrochloride was used as the control. The cell viabilities of both control and drugloaded nanoparticles were decreased with increasing incubation time. The cell viabilities of the two groups that were incubated for 24 hours were $27.6 \%$ and $31.2 \%$, and those that were incubated for 48 hours exhibited cell viabilities of $2.9 \%$ and $6.8 \%$. After 3 -day incubation, only $0.3 \%$ of cancer cells survived in the doxorubicin hydrochloride group, and the survival rate of cancer cells in the drug-loaded nanoparticles was 3.3\%. This result revealed that the drug-loaded nanoparticles could inhibit the proliferation of cancer cells efficiently, and that the in vitro antitumor efficiency of drugloaded nanoparticles was comparable to that of doxorubicin hydrochloride.

The cellular uptake of the drug-loaded nanoparticles was studied by confocal laser scanning microscopy. Both the drug-loaded nanoparticles and doxorubicin hydrochloride were incubated with 4T1 cells for 3 and 13 hours, respectively. The nuclei of the cells were dyed blue. The photographs

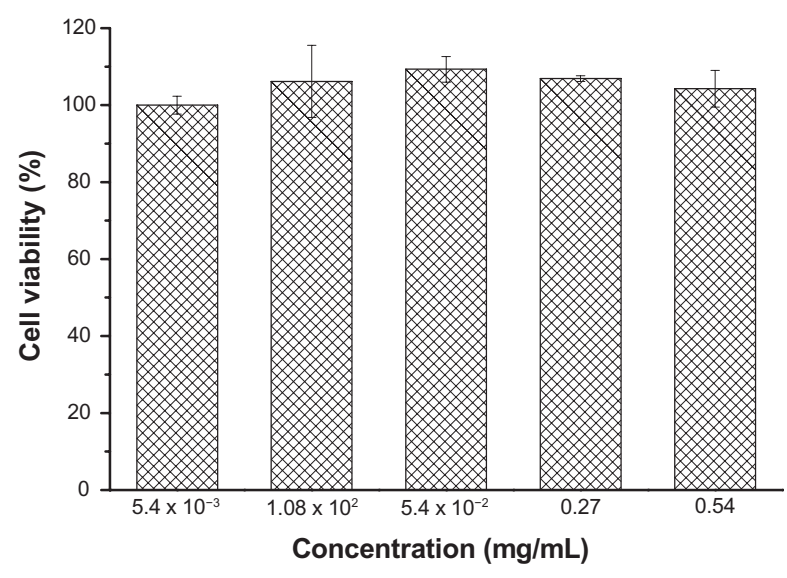

Figure 6 The cytotoxicity of blank polyrotaxane nanoparticles.

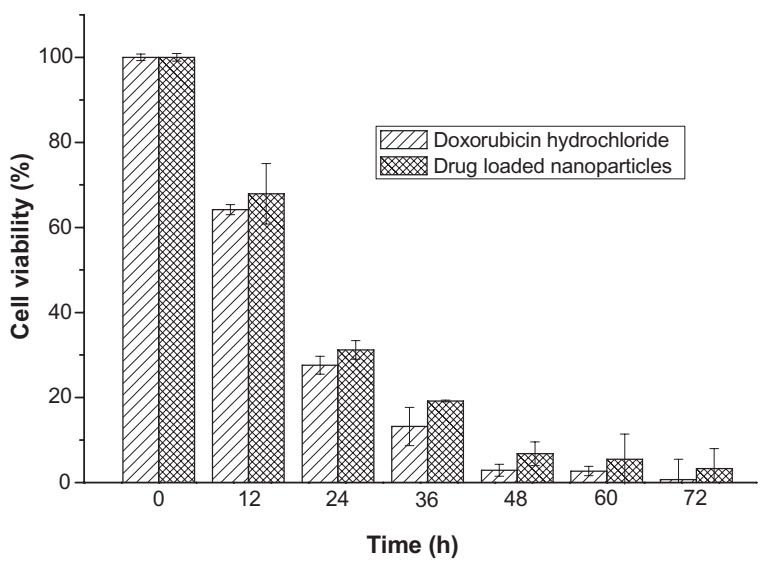

Figure 7 The in vitro inhibition effect of drug-loaded polyrotaxane nanoparticles on 4TI breast cancer cells.

Note: The concentration of doxorubicin was $10 \mu \mathrm{g} / \mathrm{mL}$.

are presented in Figure 8. In the first 3 hours of incubation, a strong red fluorescent effect was observed with doxorubicin hydrochloride in both the cytoplasm and nuclei of the cells. In the cells incubated with drug-loaded nanoparticles, red fluorescent of doxorubicin was also observed in the nuclei, but the red fluorescent in the cytoplasm was stronger than that in nuclei. After being incubated for 13 hours, the red fluorescent distribution was very different. The fluorescent strength in the nuclei of the cells treated with doxorubicin hydrochloride was weakened and the cytoplasm and nuclei were diffuse, while the nucleus membrane was not clear. In the cells treated with drug-loaded nanoparticles, the red fluorescent was strengthened in the nuclei after 13 hours of incubation.

Doxorubicin hydrochloride, a hydrophilic antitumor drug, was internalized into cells by diffusion, it diffused very quickly both from the medium to the cytoplasm and from the cytoplasm to the nuclei, thus a strong red fluorescent was observed in both the cytoplasm and the nuclei in the cells treated with doxorubicin hydrochloride for the first 3 hours. With the incubation time increasing, the drug was consumed and the cells were destroyed; thus, the strength of the fluorescent effect in the nuclei was weakened, and the cytoplasm and nuclei of the cells were diffusive. Furthermore, the nucleus membrane was not clear. The internalization of drug-loaded nanoparticles did not occur due to diffusion, so the endocytosis of drug-loaded nanoparticles was slower than that of doxorubicin hydrochloride. As a result, the fluorescent noted in the cells treated with drug-loaded nanoparticles was weak and was mainly localized in the cytoplasm of the cells within the first 3 hours. The doxorubicin was released from the nanoparticles and protonized to hydrophilic due to the acidity in endosomes and/or lysosomes, the protonized doxorubicin diffused from cytoplasm to nuclei quickly, ${ }^{32}$ thus, the 

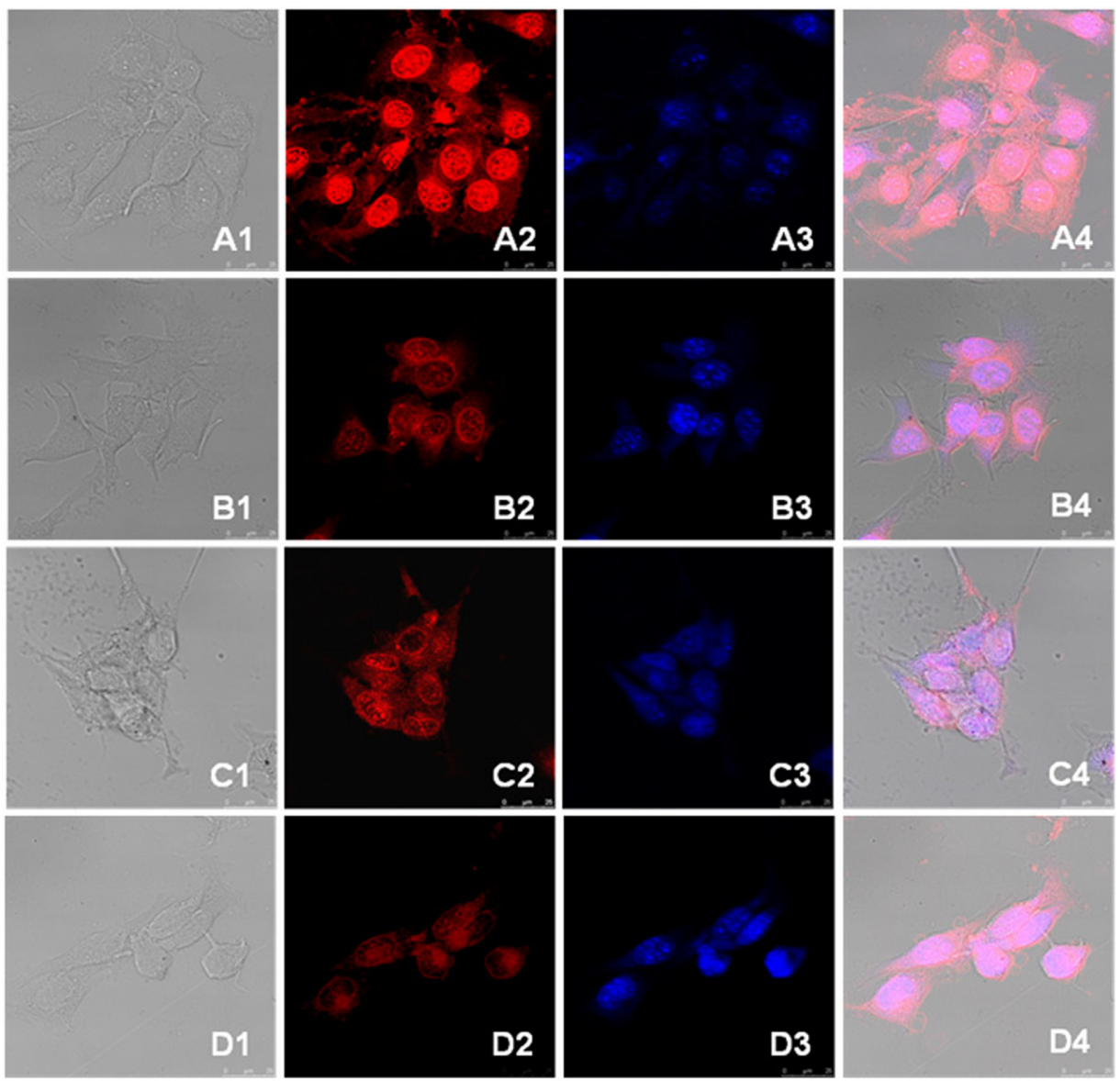

Figure 8 Confocal microscopy photographs of doxorubicin and drug-loaded nanoparticles incubated with 4TI breast cancer cells. (A) doxorubicin hydrochloride and (B) doxorubicin-loaded nanoparticles incubated for 3 hours; (C) doxorubicin hydrochloride and (D) doxorubicin-loaded nanoparticles incubated for 13 hours. Note: The photographs from left to right (numbered I to 4) are the overlapped photos of bright field and doxorubicin stained nuclei.

fluorescent in the nuclei was stronger after 13-hour incubation than that after 3-hour incubation. These results demonstrated that the trapped doxorubicin could be released from the polyrotaxane nanoparticles efficiently in cytoplasm.

A breast cancer model in mice was used to test the in vivo antitumor efficacy of the drug-loaded polyrotaxane nanoparticles. The concentration of the dose of the drug was $5 \mathrm{mg}$ per $\mathrm{kg}$ of body weight, and the drug-loaded nanoparticles were administrated by injection into the tail vein of the mice. A total of 12 mice in each group were used as parallel samples; two mice in both the blank nanoparticles and doxorubicin hydrochloride groups died during the in vivo experiments. The tumor volumes were tested, and the statistic results have been presented in Figure 9. The average volumes of tumors administrated with saline and blank nanoparticles were 1800 and $1550 \mathrm{~mm}^{3}$, respectively. The volumes of tumors treated with doxorubicin hydrochloride and drug-loaded nanoparticles were about 950 and $800 \mathrm{~mm}^{3}$. The statistical results showed a significant difference between the two groups $(P<0.05)$. Specifically, doxorubicin-loaded nanoparticles exhibited better tumor growth suppression than nanoparticles loaded with doxorubicin hydrochloride.

The in vivo tumor inhibition rate of the drug-loaded nanoparticles was calculated and the result is presented in Figure 10. The antitumor activity of the drug-loaded

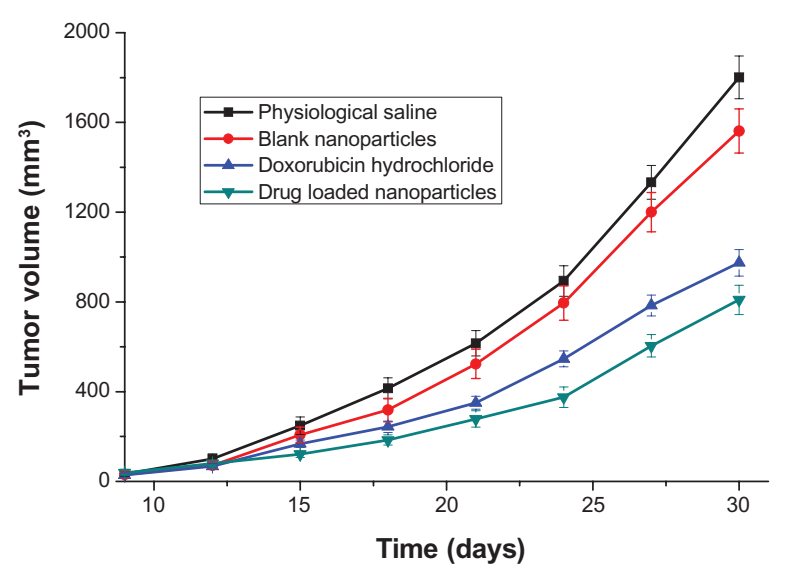

Figure 9 The in vivo inhibition effect of doxorubicin-loaded polyrotaxane nanoparticles on tumor growth in mice via venous injection. 


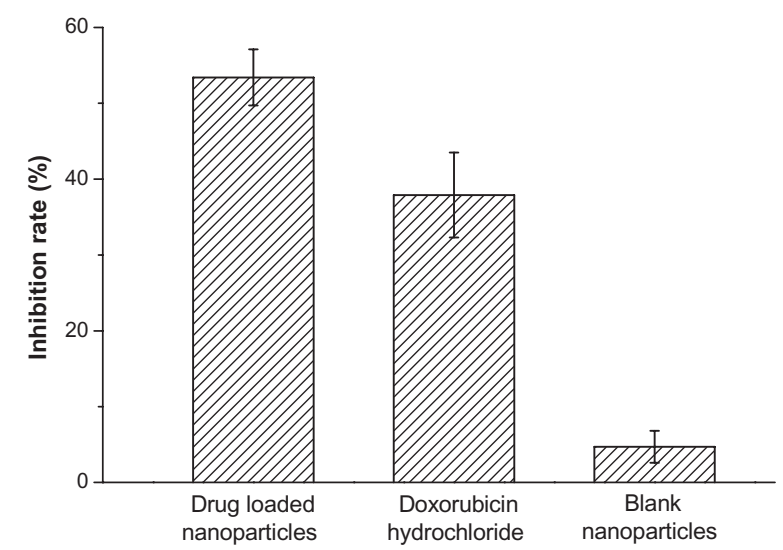

Figure 10 The inhibition rate of drug-loaded nanoparticles to breast cancer in mouse models.

nanoparticles was optimal, with its inhibition rate as high as $53 \%$; conversely, the inhibition rate of doxorubicin hydrochloride was $38 \%$. This result revealed that the in vivo inhibition efficacy of doxorubicin-loaded polyrotaxane nanoparticles was better than that of the doxorubicin hydrochloride-loaded nanoparticles, suggesting that the polyrotaxane nanoparticles were promising carriers of the drug for antitumor drug delivery.

Doxorubicin was seriously toxic to the heart of the mice in the study. ${ }^{33-35}$ A pathological examination of the drug-loaded nanoparticles was conducted. The histological photographs of cardiac muscle treated with doxorubicin hydrochloride and drug-loaded nanoparticles are presented in Figure 11. The inflammatory reaction was clearly observed, and the texture of the cardiac muscle was destroyed (Figure 11A) in the sample treated with doxorubicin hydrochloride. In contrast, this phenomenon was not observed in the photographs of the cardiac muscles of mice treated with drug-loaded nanoparticles (Figure 11B). This demonstrated that the encapsulation of polyrotaxane nanoparticles could decrease the cytotoxicity of doxorubicin.

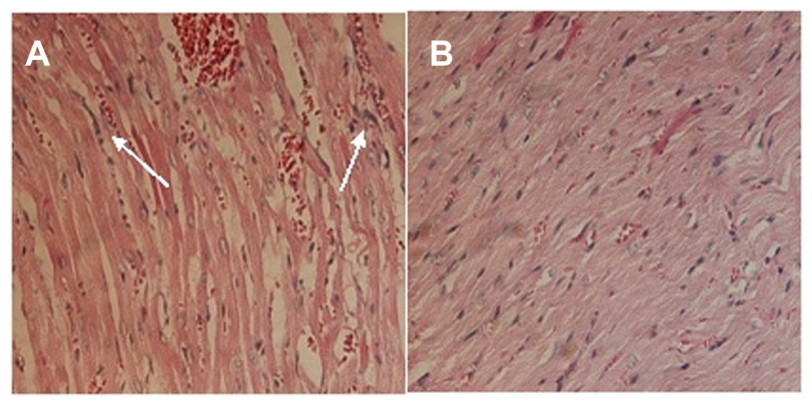

Figure II Histological photographs of mice cardiac muscles administrated with doxorubicin hydrochloride (A) and drug-loaded polyrotaxane nanoparticles (B). Note: The arrows show the inflammation.

\section{Conclusion}

A supramolecular nanoparticle generated by the self-assembly polyrotaxanes composed of $\alpha$-cyclodextrins and Cin-PEG was prepared. The morphology of the nanoparticles was changed from nanovesicle to micelle after the antitumor drug doxorubicin was loaded. The release profile investigation found that the sustained release could last for 32 hours, and the release replicated one-order release after the burst-release period. The drug-loaded nanoparticles were co-cultured with mouse 4T1 breast cancer cells to evaluate the in vitro antitumor efficacy, and the results showed that the internalization of the drugloaded nanoparticles was effective, and that the drug could be released in cytoplasm. The results of the in vivo experiment revealed that the drug-loaded polyrotaxane nanoparticles exhibited efficient antitumor activity to breast cancer cells, and this effect was better than that of the doxorubicin hydrochloride. The pathological examination demonstrated that the encapsulation of polyrotaxane nanoparticles could decrease the cardiac toxicity of doxorubicin. The polyrotaxane nanoparticle is a promising carrier for antitumor drug delivery in a wide range of biomedical applications.

\section{Acknowledgments}

This research work was supported by the National Basic Research Program of China (National 973 program, No 2011CB606206), the National Science Foundation of China (NSFC, No 31070849, 50830105, 51133004), the Program for New Century Excellent Talents in University, Ministry of Education (MOE, NCET-10-0564), the International Collaboration Project of Ministry of Science and Technology (MOST, No 2010DFA51550), the Program for Changjiang Scholars, and the Innovative Research Team in University (IRT1163).

\section{Disclosure}

The authors report no conflicts of interest in this work.

\section{References}

1. Lavasanifar A, Samuel J, Kwon GS. Poly(ethylene oxide)-blockpoly(L-amino acid) micelles for drug delivery. Adv Drug Deliv Rev. 2002;54(2):169-190.

2. Li SD, Huang L. Stealth nanoparticles: high density but sheddable PEG is a key for tumor targeting. $J$ Control Release. 2010;145(3):178-181.

3. Ranganathan R, Madanmohan S, Kesavan A, et al. Nanomedicine: towards development of patient-friendly drug-delivery systems for oncological applications. Int J Nanomedicine. 2012;7:1043-1060.

4. Wang K, Liu L, Zhang T, et al. Oxaliplatin-incorporated micelles eliminate both cancer stem-like and bulk cell populations in colorectal cancer. Int J Nanomed. 2011;6:3207-3218.

5. Cai LL, Liu P, Li X, et al. RGD peptide-mediated chitosan-based polymeric micelles targeting delivery for integrin-overexpressing tumor cells. Int J Nanomedicine. 2011;6:3499-3508. 
6. Lee ES, Na K, Bae YH. Doxorubicin loaded $\mathrm{pH}$-sensitive polymeric micelles for reversal of resistant MCF-7 tumor. J Control Release. 2005;103(3):405-418

7. Yu D, Peng P, Dharap SS, et al. Antitumor activity of poly(ethylene glycol)-camptothecin conjugate: the inhibition of tumor growth in vivo. J Control Release. 2005;110(1):90-102.

8. Tong R, Cheng J. Paclitaxel-initiated, controlled polymerization of lactide for the formulation of polymeric nanoparticulate delivery vehicles. Angew Chem Int Ed Engl. 2008;47(26):4830-4834.

9. Greenwald RB, Choe YH, McGuire J, Conover CD. Effective drug delivery by PEGylated drug conjugates. Adv Drug Deliv Rev. 2003; 55(2):217-250.

10. Tang S, Huang X, Chen X, Zheng N. Hollow mesoporous zirconia nanocapsules for drug delivery. Adv Funct Mater. 2010;20(15): 2442-2447.

11. Liu Z, Fan AC, Rakhra K, et al. Supramolecular stacking of doxorubicin on carbon nanotubes for in vivo cancer therapy. Angew Chem Int Ed Engl. 2009;48(41):7668-7672.

12. Liu Z, Robinson JT, Sun XM, Dai HJ. PEGylated nanographene oxide for delivery of water-insoluble cancer drugs. J Am Chem Soc. 2008;130(33):10876-10877.

13. Wang J, Jiang M. Polymeric self-assembly into micelles and hollow spheres with multiscale cavities driven by inclusion complexation. J Am Chem Soc. 2006;128(11):3703-3708.

14. Wang Y, Ma N, Wang Z, Zhang X. Photocontrolled reversible supramolecular assemblies of an azobenzene-containing surfactant with alpha-cyclodextrin. Angew Chem Int Ed Engl. 2007;46(16): 2823-2826.

15. Ohga K, Takashima Y, Takahashi H, Kawaguchi Y, Yamaguchi H, Harada A. Preparation of supramolecular polymers from a cyclodextrin dimer and ditopic guest molecules: control of structure by linker flexibility. Macromolecules. 2005;38(14):5897-5904.

16. Liu Y, Ke CF, Zhang HY, Cui J, Ding F. Complexation-induced transition of nanorod to network aggregates: alternate porphyrin and cyclodextrin arrays. J Am Chem Soc. 2008;130(2):600-605.

17. Rusa CC, Bullions TA, Fox J, Porbeni FE, Wang X, Tonelli AE. Inclusion compound formation with a new columnar cyclodextrin host. Langmuir. 2002;18(25):10016-10023.

18. Topchieva IN, Tonelli AE, Panova IG, et al. Two-phase channel structures based on alpha-cyclodextrin-polyethylene glycol inclusion complexes. Langmuir. 2004;20(21):9036-9043.

19. Harada A, Kamachi M. Complex formation between poly(ethylene glycol) and $\alpha$-cyclodextrin. Macromolecules. 1990;23(10):2821-2823.

20. Li J, Ni X, Zhou Z, Leong KW. Preparation and characterization of polypseudorotaxanes based on block-selected inclusion complexation between poly(propylene oxide)-poly(ethylene oxide)-poly(propylene oxide) triblock copolymers and alpha-cyclodextrin. J Am Chem Soc. 2003;125(7):1788-1795.

21. Ooya T, Choi HS, Yamashita A, et al. Biocleavable polyrotaxaneplasmid DNA polyplex for enhanced gene delivery. J Am Chem Soc. 2006;128(12):3852-3853.
22. Li J, Yang C, Li HZ, et al. Cationic supramolecules composed of multiple oligoethylenimine-grafted $\beta$-cyclodextrins threaded on a polymer chain for efficient gene delivery. Adv Mater. 2006;18(22):2969-2974.

23. Ren L, Ke F, Chen Y, Liang D, Huang J. Supramolecular ABA triblock copolymer with polyrotaxane as B block and its hierarchical selfassembly. Macromolecules. 2008;41(14):5295-5300.

24. Huang J, Ren L, Chen Y. pH-/temperature-sensitive supramolecular micelles based on cyclodextrin polyrotaxane. Polym Int. 2008;57(5): 714-721.

25. Tsai CC, Leng S, Jeong KU, et al. Supramolecular structure of $\beta$-cyclodextrin and poly(ethylene oxide)-block-poly(propylene oxide)block-poly(ethylene oxide) inclusion complexes. Macromolecules. 2010;43(22):9454-9461.

26. Shuai X, Ai H, Nasongkla N, Kim S, Gao J. Micellar carriers based on block copolymers of poly(epsilon-caprolactone) and poly(ethylene glycol) for doxorubicin delivery. J Control Release. 2004;98(3): 415-426.

27. Harada A, Li J, Kamachi M. The molecular necklace: a rotaxane containing many threaded $\alpha$-cyclodextrins. Nature. 1992;356(6367):325-327.

28. Harada A, Li J, Kamachi M. Preparation and properties of inclusion complexes of polyethylene glycol with .alpha.-cyclodextrin. Macromolecules. 1993;26(21):5698-5703.

29. Uyar T, Hunt MA, Gracz HS, Tonelli AE. Crystalline cyclodextrin inclusion compounds formed with aromatic guests: guest-dependent stoichiometries and hydration-sensitive crystal structures. Cryst Growth Des. 2006;6(5):1113-1119.

30. Miyauchi M, Hoshino T, Yamaguchi H, Kamitori S, Harada A. A [2]rotaxane capped by a cyclodextrin and a guest: Formation of supramolecular [2]rotaxane polymer. J Am Chem Soc. 2005;127: 2034-2035.

31. Miyauchi M, Takashima Y, Yamaguchi H, Harada A. Chiral supramolecular polymers formed by host-guest interactions. J Am Chem Soc. 2005;127(9):2984-2989.

32. Liu R, Li D, He B, et al. Anti-tumor drug delivery of $\mathrm{pH}$-sensitive poly(ethylene glycol)-poly(L-histidine-)-poly(L-lactide) nanoparticles. $J$ Control Release. 2011;152(1):49-56.

33. Tan ML, Friedhuber AM, Dunstan DE, Choong PF, Dass CR. The performance of doxorubicin encapsulated in chitosan-dextran sulphate microparticles in an osteosarcoma model. Biomaterials. 2010;31(3): 541-551.

34. Xiao K, Luo J, Li Y, Lee JS, Fung G, Lam KS. PEG-oligocholic acid telodendrimer micelles for the targeted delivery of doxorubicin to B-cell lymphoma. J Control Release. 2011;155(2):272-281.

35. Mukhopadhyay P, Batkai S, Rajesh M, et al. Pharmacological inhibition of CB1 cannabinoid receptor protects against doxorubicin-induced cardiotoxicity. J Am Coll Cardiol. 2007;50(6):528-536.
International Journal of Nanomedicine

\section{Publish your work in this journal}

The International Journal of Nanomedicine is an international, peerreviewed journal focusing on the application of nanotechnology in diagnostics, therapeutics, and drug delivery systems throughout the biomedical field. This journal is indexed on PubMed Central, MedLine, CAS, SciSearch $\AA$, Current Contents ${ }^{\circledR} /$ Clinical Medicine,

\section{Dovepress}

Journal Citation Reports/Science Edition, EMBase, Scopus and the Elsevier Bibliographic databases. The manuscript management system is completely online and includes a very quick and fair peer-review system, which is all easy to use. Visit http://www.dovepress.com/ testimonials.php to read real quotes from published authors. 